\title{
Use of Platelet Rich Fibrin in Acute Traumatic Tympanic Membrane Perforation Compared With the Conservative Treatment
}

\author{
Mohamed F. Shindy ${ }^{a}$, Ola S. El-Shimi ${ }^{b}$, Mahmoud S. Belal ${ }^{c}$, Abdelrahman A. Abdelalim ${ }^{\text {a }}$ \\ Department of \\ Otorhinolaryngology, Benha \\ Faculty of Medicine, Benha \\ University, Egypt. \\ Department of Clinical and \\ Chemical Pathology, Faculty \\ of Medicine, Benha \\ University, Egypt, \\ Department \\ Otorhinolaryngology, Naser \\ institute, Cairo, Egypt. \\ Correspondence to: \\ Abdelrahman A. Abdelalim, \\ department of \\ Otorhinolaryngology, Benha \\ Faculty of Medicine, Benha \\ University, Egypt \\ Email: \\ aborazan2009@gmail.com \\ Received: 3 December 2019 \\ Abstract: \\ Objectives: To evaluate the effect of platelet rich fibrin in treating \\ acute traumatic tympanic membrane perforation as regards wound \\ healing and hearing outcome compared with conservative treatment. \\ Methods: This study was conducted on 50 patients presented with \\ acute traumatic tympanic membrane perforation classified randomly \\ into 2 groups: the study group; (25 patients) treated with platelet rich \\ fibrin membrane and control group; (25 patients) underwent \\ conservative treatment. Patients were followed up for 6 weeks to \\ assess healing rate of the tympanic membrane perforation and hearing \\ improvement. Results: In the group where platelet rich fibrin was \\ used, complete perforation closure occurred in $88 \%$ of patients, while \\ in the group treated conservatively, closure was obtained in $56 \%$ (P \\ 0.004). The mean air bone gap improved in the platelet rich fibrin- \\ treated group from $18.83 \pm 3.99 \mathrm{~dB}$ to $3.38 \pm 4.13 \mathrm{~dB}$ and in the control \\ Accepted:19 January 2020 \\ group from $18.90 \pm 3.43 \mathrm{~dB}$ to $9.84 \pm 8.04 \mathrm{~dB}$. The post-treatment mean \\ air bone gap significantly improved better in the platelet rich fibrin- \\ treated group than in the control group (P 0.004). Hearing gain by $>10$ \\ $\mathrm{dB}$ was obtained in $88 \%$ of patients in the platelet rich fibrin group \\ and in $44 \%$ of patients in the control group (P 0.001). Conclusion: In \\ comparison with the conservative management, platelet rich fibrin, as \\ an autologus simple graft method, provided more healing rates with \\ better audiological outcomes with low cost and no complications.
}

Keywords: Platelet rich fibrin; Traumatic perforation; Tympanic membrane perforation. 



\section{Introduction}

Tympanic membrane (TM) perforation is one of the commonest causes of conductive hearing loss; in this case, tympanic membrane integrity must be re-established quickly to restore hearing and to protect the middle ear from external insults [1].

Traumatic tympanic membrane perforation is an injury of the eardrum, which is commonly faced by otolaryngologists. Its causes include, sudden increase in ear pressure, chemical burns, direct penetrating trauma, or barotrauma [2]. Usually acute tympanic membrane perforations (TMPs) heal within 7-10 days [3]. About 10-20\% of TMPs may not heal within 3 months and become chronic [4].

Many different materials have previously been used to treat traumatic TMPs, including heparin, hyaluronic acid, fibroblast growth factor, epidermal growth factor, platelet-derived growth factor or transforming growth factor [5]. Also other materials have been used, such as gel foam, paper patch, and autograft to provide stromal support, silver nitrate and trichloride acetic acid cauterization to refresh the perforation edges, and oil to enhance cellular regeneration and mobilization [6].
Platelet concentrates have been used extensively by plastic surgeons, maxillofacial surgeons, and orthopedic surgeons to enhance wound healing. Platelet-rich plasma (PRP) and platelet-rich fibrin (PRF) are different platelet concentrates that have been studied [7].

PRF differs from PRP that there is no synthetic material or anticoagulant is added; it has low costs and can be obtained easily in a short time. It is noteworthy that this technique has been the easiest and cheapest method of obtaining blood products [8].

\section{Materials and methods}

This case-control study was conducted at Naser Institute for Research and Treatment, Cairo, Egypt; during the period from October 2018 till August 2019. The study protocol was approved by the local ethical committee of Faculty of Medicine, Benha University, Egypt. All patients gave their written informed consent to participate.

Fifty patients presented with acute traumatic TM perforation had been selected from the outpatient clinic, with traumatic perforation within the previous 7 days and showing an air bone gap $(\mathrm{ABG}) \leq 25 \mathrm{~dB}$. 
Patients with history of hearing loss before the trauma, previous ear surgeries, traumatic perforations more than 7 days, associated with other otological problems, infected cases at time of presentation and/or patients with $A B G>25 \mathrm{~dB}$ were excluded from the study. Patients with history of coagulopathies or taking anticoagulants within the previous 10 days were excluded as well.

All patients in this study underwent a thorough clinical assessment including; complete history taking, full otorhinolaryngological examination and otoendoscopic examination of the ear drum, using $4 \mathrm{~mm} 0^{\circ}$ endoscope attached to scope camera and recording system. Images were taken and analysed using IMAGEJ software package [Figure 1].

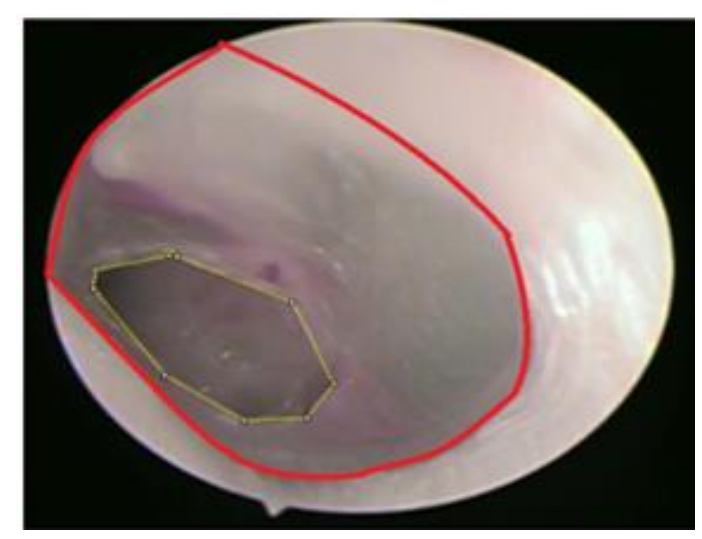

Figure 1: Image of a medium-sized traumatic TM perforation

The size of TM perforation had been determined and graded according to Saliba classification [9]: Grade I: small, less than $25 \%$ of the tympanic membrane surface, Grade II: medium, $25-50 \%$ of the tympanic membrane surface, Grade III: large, 50$75 \%$ of the tympanic membrane surface and Grade IV: subtotal, more than $75 \%$ of the tympanic membrane surface.

An initial Pure Tone Audiometry (PTA) was performed at time of presentation and $\mathrm{ABG}$ was calculated as the mean of $\mathrm{ABG}$ at three frequencies (500, 1000 and $2000 \mathrm{~Hz}$ ). All patients were treated in an outpatient setting in a sterile environment. We had randomly selected 25 patients to be treated with platelet rich fibrin (PRF-treated group) and 25 patients to be treated conservatively (control group).

In PRF-treated group, a $10 \mathrm{ml}$ venous blood sample was drawn from each patient into sterile, dry, glass vacuum plain tubes (without anticoagulant). Tubes were centrifuged immediately for $12 \mathrm{~min}$ at 2700-3000 rpm. Three layers were obtained; a top layer of platelet poor plasma, a middle layer of PRF clot and a basal layer of RBCs.

The PRF was removed from the tube cautiously with a sterile forceps and stripped from the adjacent red blood cell layer and the PRF serum was absorbed into 
a gauze pad to obtain a highly resistant membrane rich in fibrin [Figure 2].

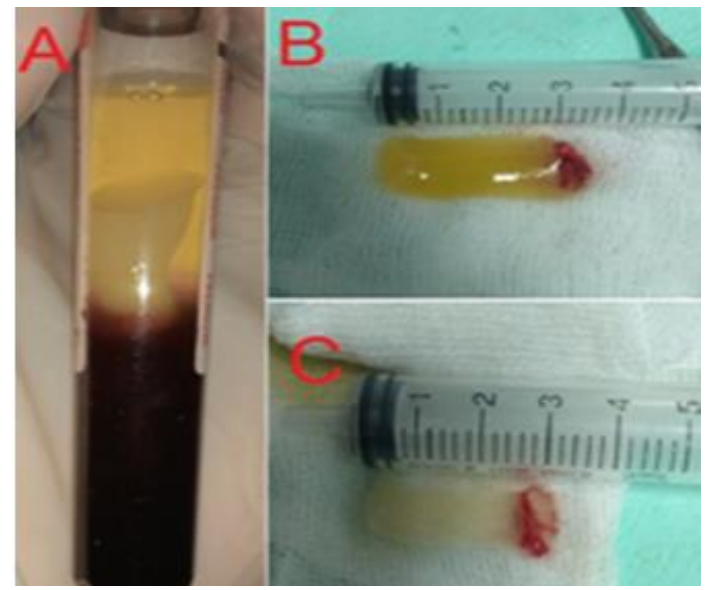

Figure 2: PRF preparation; A: whole blood separation layers, B: Separated PRF clot and C: PRF membrane after preparation.

The patient was positioned supine with the affected ear turned upwards. Local anesthesia, $10 \%$ Xylocaine was used to anesthetize the tympanic membrane by placing soaked cotton ball into the external auditory canal wall over the surface of the tympanic membrane for about $10 \mathrm{~min}$ then removed.

Autologous PRF membrane was cut to a size approximately 1.5 times larger than the perforation size. It was plugged through the perforation site, without using any middle ear packing materials [Figure 3].

All patients were advised to avoid ear canal instrumentation, forceful coughing, sneezing or blowing of nose and keeping the ear dry. Follow up visits at the outpatient clinic were scheduled after 1, 2 and 6 weeks. After 6 weeks
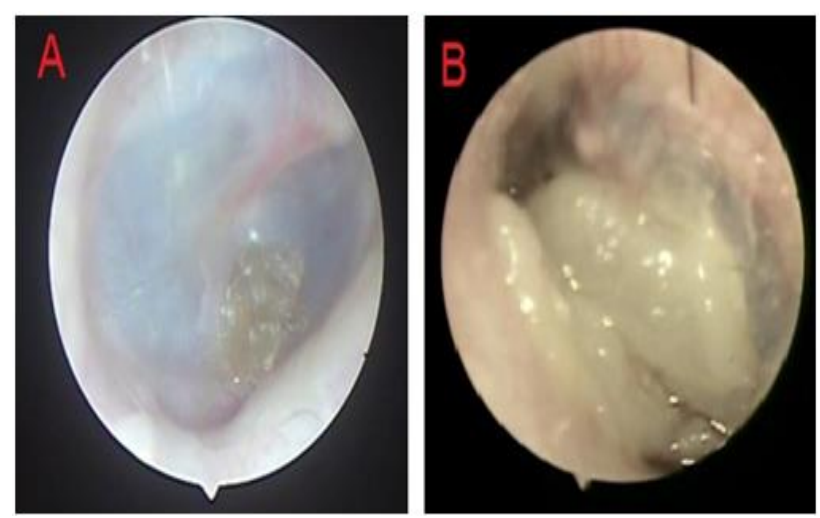

Figure 3: PRF application in small sized perforation (A) and in large sized perforation (B).

All patients were subjected to an endoscopic examination with the same technique using $4 \mathrm{~mm} 0^{\circ}$ endoscope attached to scope camera and recording system, PTA at the same audiological center and ABG measurement. Hearing gain was calculated as the difference between preand post-treatment $\mathrm{ABG}$ means.

Obtained data were statistically analyzed using SPSS version 16 software (SPSS Inc, Chicago, IL, USA). Categorical data were presented as number (percentage) and chi square test was used to analyze them. While quantitative data were expressed as mean \pm standard deviation (SD) and Student's ' $t$ " test, was used to analyze them. P-value $\leq 0.05$ was considered the accepted level of significance in this work. 


\section{Results}

The study group (PRF-treated) consisted of 25 patients; 5 males and 20 females aged 8-44 years (mean age 25.32 \pm 9.66 years). While the control group (conservativelytreated) consisted of 25 patients; 8 males and 17 females aged 7-42 years (mean age $25.48 \pm 10.14$ years). Both groups were ageand sex-matched.

The etiology of the perforation was slap injury in 35 patients $(70 \%)$, iatrogenic injury in 7 patients (14\%) and self-induced in 8 patients $(16 \%)$ of the total patients included in the study.

Patients were classified according to Saliba classification [9] based on the TMP size. In PRF-treated group; 9 patients (36\%) had grade I (small-sized TMP), 11 patients (44\%) had grade II (medium-sized TMP), 5 patients $(20 \%)$ had grade III (large-sized TMP) and no patients had Grade IV (subtotal TMP). While, in the control group; 8 patients $(32 \%)$ had grade I TMP, 13 patients $(52 \%)$ had grade II TMP, 4 patients $(16 \%)$ had grade III TMP and no patients had Grade IV TMP. No significant differences were observed between the two groups regarding perforation size.

All patients were examined after 1 and 2 weeks [Figure 4]. There were two infected

case in the PRF-treated group and three infected cases in the control group. They received proper treatment and full cure was achieved without graft disruption.

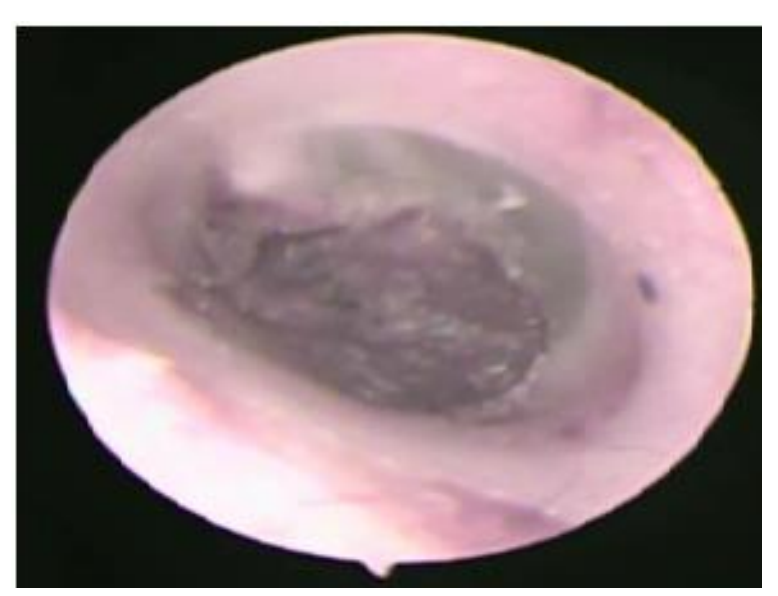

Figure 4: An image of PRF applied to large TM perforation after 1 week.

On examination after 6 weeks, complete healing of the perforated TM in the PRFtreated group was achieved in grade I by $100 \%$, in grade II by $90.9 \%$ and in grade III by $60 \%$ compared with the control group; where complete healing of the perforated TM was achieved in grade I by $87.5 \%$, in grade II by $53.8 \%$ and in grade III by $0 \%$. PRF-treated patients with grades II and III perforation size significantly achieved complete healing more than the conservatively-treated patients (P 0.047 and 0.05 respectively) while no difference was observed in grade I patients [Table 1]. 
The pre-treatment mean ABG didn't show any differences between the studied groups [Table 2].

However, the post-treatment mean $\mathrm{ABG}$ was significantly reduced in grades II and III of PRF-treated group rather than the conservatively-treated group (P 0.003 and 0.005 respectively), while no difference in post-treatment mean $\mathrm{ABG}$ was observed in grade I patients [Table 3].

Comparing pre- and post-treatment mean $\mathrm{ABG}$ in PRF-treated group revealed that the mean ABG significantly decreased after treatment by PRF in all TMP grades [Chart 1].
While in control group, the mean $A B G$ significantly decreased post-conservative treatment in grades I and II only [Chart 2].

In order to evaluate hearing improvement, hearing gain was assessed. It was found that the mean hearing gain was significantly increased in the PRF-treated group than that in the control group (P 0.002). Moreover, by classifying hearing gain either $>10 \mathrm{~dB}$ or $\leq 10$ $\mathrm{dB}$, it was observed that hearing gain $>10 \mathrm{~dB}$ was significantly more frequent in the PRFtreated group, 22 patients $(88 \%)$ compared with 11 patients $(44 \%)$ in the control group (P 0.001) [Table 4].

Table 1: Comparison of the healing achievement between studied groups

\begin{tabular}{llccc}
\hline & & PRF-treated group & $\begin{array}{c}\text { Conservatively-treated } \\
\text { group }\end{array}$ & P \\
\hline Complete & Grade I & $9 / 9(100 \%)$ & $7 / 8(87.5 \%)$ & 0.274 \\
healing & Grade II & $10 / 11(90.9 \%)$ & $7 / 13(53.8 \%)$ & 0.047 \\
& Grade III & $3 / 5(60 \%)$ & $0 / 4(0 \%)$ & 0.050 \\
\hline Total & & $22 / 25(88 \%)$ & $14 / 25(56 \%)$ & 0.004 \\
\hline
\end{tabular}

Data expressed as number \& percentage within group

Table 2: Comparison of pre-treatment ABG between studied groups

\begin{tabular}{|c|c|c|c|c|}
\hline & & PRF-treated group & $\begin{array}{c}\text { Conservatively-treated } \\
\text { group }\end{array}$ & $\mathbf{P}$ \\
\hline \multirow{3}{*}{$\begin{array}{l}\text { Pre-treatment } \\
\text { ABG }\end{array}$} & Grade I & $14.41 \pm 1.43$ & $15.17 \pm 1.37$ & 0.281 \\
\hline & Grade II & $19.96 \pm 1.47$ & $19.45 \pm 1.41$ & 0.398 \\
\hline & Grade III & $24.32 \pm 0.93$ & $24.57 \pm 0.85$ & 0.685 \\
\hline Total & & $18.83 \pm 3.99$ & $18.90 \pm 3.43$ & 0.949 \\
\hline
\end{tabular}


Table 3: Comparison of post-treatment ABG between studied groups

\begin{tabular}{rrccc}
\hline \hline & & PRF-treated group & $\begin{array}{c}\text { Conservatively-treated } \\
\text { group }\end{array}$ & P \\
\hline \multirow{2}{*}{ Post-treatment } & Grade I & $1.66 \pm 2.5$ & $3.75 \pm 5.82$ & 0.343 \\
ABG & Grade II & $2.86 \pm 3.56$ & $10.49 \pm 6.94$ & 0.003 \\
& Grade III & $7.64 \pm 5.32$ & $19.95 \pm 3.04$ & 0.005 \\
\hline & Total & $3.38 \pm 4.13$ & $9.84 \pm 8.04$ & 0.004 \\
\hline
\end{tabular}

Data expressed as mean $\pm \mathrm{SD}$

Table 4: Comparison of hearing gain in studied groups:

\begin{tabular}{ccccc}
\hline & & PRF-treated group & Conservatively-treated group & P \\
\hline Hearing gain & & $15.44 \pm 3.89$ & $9.05 \pm 6.39$ & 0.002 \\
\hline \multirow{2}{*}{ Hearing gain } & $>\mathbf{1 0 d B}$ & $22(88 \%)$ & $11(44 \%)$ & 0.001 \\
& $\leq \mathbf{1 0} \mathbf{~ d B}$ & $3(12 \%)$ & $14(56 \%)$ & \\
\hline
\end{tabular}

Data expressed as mean \pm SD and number (percentage)

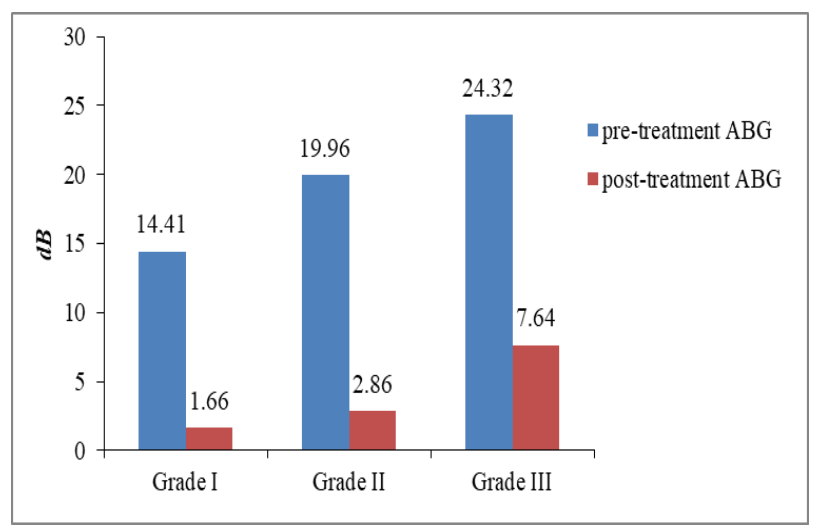

Chart 1: The difference between pre- and post-treatment $\mathrm{ABG}$ in PRF-treated group

\section{Discussion}

The TM is an important component of sound conduction as its vibratory characteristic is necessary for sound transmission in human beings [10]. Traumatic TMP treatment ranges from

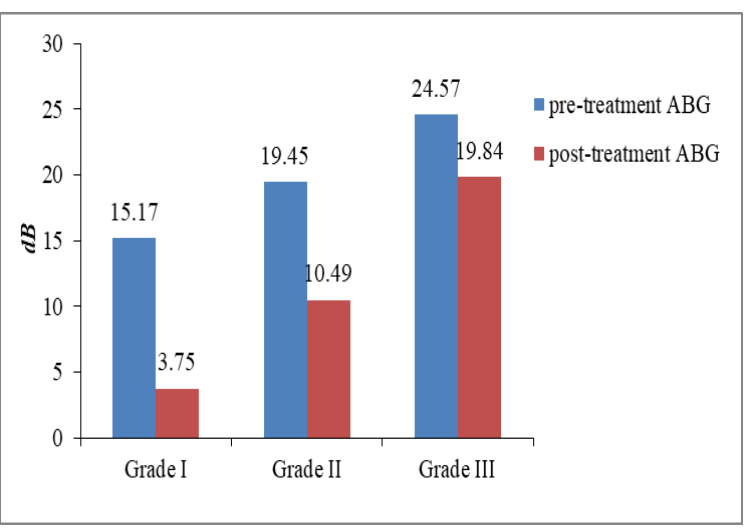

Chart 2: The difference between pre- and posttreatment $\mathrm{ABG}$ in control group

inactive watchful waiting, active intervention to surgical intervention [11]. Although the closure rates of TM perforation do not vary greatly, some otolaryngologists prefer immediate procedures to accelerate its healing [12]. 
These procedures include the use of early patching and surgical intervention, Silastic sheet, and biomaterials [13]. Autogenous materials can be used for tympanic membrane perforations as they are less invasive, cheaper, and readily obtained [14]. Platelet rich fibrin (PRF) had been described and used for repair and regeneration of different tissues by Choukroun et al., [15] who firstly introduced its clinical application in oral, maxillofacial and implant surgery.

PRF membrane can be easily adjusted by cutting to size. It is elastic and can be easily adapted as graft material during use [16]. PRF membrane offers both mechanical and inflammatory protection to the graft with no undesirable tissue reaction, it may be an ideal patching material in the treatment of traumatic TM perforation [14].

In the literature, there are few experimental and clinical studies discussing the role of platelet rich fibrin in the repair of traumatic tympanic membrane perforation. Habesoglu et al., [17] studied the effects of platelet rich fibrin for the healing of acute tympanic membrane perforation, in that study they observed the closure rates and time without the audiological outcomes. Gur et al., [14] compared the use of a PRF membrane versus the use of paper patch as regards healing time, recovery rates, and the mean air bone gap correction. Experimentally, Ensari et al., [16] evaluated the effect of platelet rich fibrin membrane in the repair of perforated tympanic membrane in rats. A pilot study by Kumar [18] discussed the efficacy of platelet rich fibrin (PRF) myringoplasty technique combined with Trichloroacetic acid (TCA) chemical cauterization in the healing of traumatic tympanic membrane perforations.

PRF was used as adjuvant with tympanoplasty techniques as in a randomized controlled trial by Nair et al., [19] who studied safety and efficacy of autologous platelet rich fibrin on graft uptake in myringoplasty combining the use of PRF with temporalis fascia graft. While, Hosam et al., [20] studied the effect of topical use of platelet rich fibrin in endoscopic repair of central perforation using inlay butterfly cartilage myringoplasty.

In our study we compared the effect of platelet rich fibrin (PRF) in treating acute traumatic tympanic membrane perforation as regards wound healing and hearing outcome compared with conservative treatment. The studied groups were matched in age and sex. In our study, the 
age of patients ranged from 7 to 44 years with a mean age of 25.4 years, this matches with Gür et al., [14] who reported that the mean age of patients with traumatic TM perforation was 28.9 years. Lindeman et al., [21] also reported a mean age of 24.1 years.

Traumatic TMP occurred more among females in our study with male to female involvement ( $26 \%$ to $74 \%$ respectively). Sarojamma et al., [22] reported, as well, that the incidence of traumatic TM perforation is more among females (58\%).

Our study showed a significant higher healing rate in the PRF-treated group (88\%) more than the group treated conservatively $(56 \%)(\mathrm{P}=0.004)$. This goes in line with results of Habesoglu et al., [17] who reported that the use of PRF in acute traumatic TM perforations provided significantly better healing rates and times in comparison with spontaneous healing. In that study, the ratio of total closure of ear drum in (group 1), in which PRF was used, was $64.3 \%$, but only $22.2 \%$ of patients had total closure in (group 2), where no intervention was taken, at the end of the first month $(\mathrm{P}<0.05)$. Also, Our results match with Gür et al., [14] who compared the success rates between the use of PRF membrane (group 1) and the use of paper patch method (group 2) in the repair of traumatic TM perforations. They noticed that closure was obtained in $28(93 \%)$ perforations in (group 1) and 25 (83\%) perforations in (group 2) ( $\mathrm{P}>0.05)$.

Healing results in the PRF-treated group in our study matched with the pilot study conducted by Kumar [18] who combined PRF myringoplasty technique with Trichloroacetic acid (TCA) chemical cauterization in the treatment of traumatic tympanic membrane perforations; closure was obtained in 23 patients $(92 \%)$ out of 25 patient included in his study.

Our results regarding the healing rate in PRF-treated group came -to some extent- in line with Ensari et al., [16] who used PRF in animal experiment and reported that all the perforations of the tympanic membranes treated with PRF were closed while there were two permanent tympanic membrane perforations in the control group where healing was left to be spontaneous.

As regards the size of perforation, in the PRF-treated group our results showed that complete healing of TM perforation was achieved in grade I (small-sized) by $100 \%$, in grade II (medium-sized) by $90.9 \%$ and in grade III (large-sized) by $60 \%$. While, in the control group, complete healing of TM perforation was achieved in grade I (small- 
sized) by $87.5 \%$,in grade II (medium-sized) by $53.8 \%$ and in grade III (large-sized) by $0 \%$. This healing rate was statistically significant between both groups in both medium-sized and large-sized perforations.

In the current study, no significant difference was observed between both groups regarding pre-treatment mean $\mathrm{ABG}$. However, post-treatment mean $\mathrm{ABG}$ significantly improved in the PRF-treated group (from 18.83 to $3.38 \mathrm{~dB}$ ) more than in the control group (from 18.90 to $9.84 \mathrm{~dB}$ ) $(\mathrm{P}=0.004)$. We found that the mean $\pm \mathrm{SD}$ of hearing gain significantly improved in PRF-treated patients $(15.44 \pm 3.89 \mathrm{~dB})$ than in conservatively-treated patients $(9.05 \pm 6.39 \mathrm{~dB})(\mathrm{P}=0.002)$. This goes in line with Gür et al., [14] who reported that the improvement in the mean air bone gap was $14.1 \mathrm{~dB}$ in group 1 where PRF used. The results show that the use of PRF provides a better audiological results.

Our results showed that hearing gain by $>10 \mathrm{~dB}$ was more frequent in the PRFtreated group (22/25 patients) than in conservatively-treated group (11/25 patients). This differs from results obtained by Hosam et al., [20] who reported that hearing gain ( $\geq 10 \mathrm{~dB}$ ) was achieved in $80 \%$ of patients in group A (PRF used), which is slightly better than the hearing gain achieved in group B 60\% (PRF not used), without any significant difference $(\mathrm{P}=0.217)$. In that study, they used PRF combined with cartilage graft tympanoplasty in chronic perforations, it appeared that there was no effect of application of PRF on hearing gain as only the taken graft cases were compared.

Audiological evaluation after 6 weeks in the PRF group in our study matches with the pilot study by Kumar [18] in which the mean of preoperative $\mathrm{ABG}$ was $20.2 \mathrm{~dB}$ and post-operatively, the mean improvement in the $\mathrm{ABG}$ was $12.1 \mathrm{~dB}$ by the second month $(\mathrm{P}<0.05)$.

\section{Conclusion}

The results of our comparative study suggest that using platelet rich fibrin may offer benefits over the conservative treatment of acute traumatic tympanic membrane perforation. We observed significant better healing rates and audiological improvements by using platelet rich fibrin over the conservative treatment in medium- and large-sized traumatic tympanic membrane perforations after 6 weeks.

\section{Conflict of interest}

The authors declare no conflict of interest. 


\section{References}

1- Guneri EA, Tekin S, Yilmaz O, Ozkara E, Erdag TE, Ikiz AO, et al: The effects of hyaluronic acid, epidermal growth factor, and mitomycin in an experimental model of acute traumatic tympanic membrane perforation. Otol Neurotol 2003;24:371-6.

2- Van Rijswijk JB and Dubach P: Binaural tympanic-membrane perforations after blast injury. N Engl J Med.2017;376(19):e41.

3- Teh BM, Marano RJ, Shen Y, Friedland PL, Dilley RJ and Atlas MD : Tissue engineering of the tympanic membrane. Tissue Eng Part B Rev 2013;19:116-32.

4- Shen Y, Guo Y, Wilczynska M, Li J, Hellström S and Ny T: Plasminogen initiates and potentiates the healing of acute and chronic tympanic membrane perforations in mice. J Transl Med. 2014;12:5.

5- Ozturk K, Yaman H, Cihat AM, Arbag H, Keles $B$ and Uyar Y: Effectiveness of MeroGel hyaluronic acid on tympanic membrane perforations. Acta Otolaryngol 2006; 126:115863.

6- Santhi T and Rajan KV: A study of closure of tympanic membrane perforations by chemical cauterisation. Indian J Otolaryngol Head Neck Surg.2012; 64:389-92.

7- Choukroun JI, Braccini F, Diss A, Giordano G, Doglioli P and Dohan DM: Influence of platelet rich fibrin (PRF) on proliferation of human preadipocytes and tympanic keratinocytes: A new opportunity in facial lipostructure
(Coleman's technique) and tympanoplasty. Rev Laryngol OtolRhinol (Bord) 2007; 128(1-2):2732.

8- Dohan DM, Rasmusson L and Albrektsson T: Classification of platelet concentrates from pure platelet- rich plasma (P-PRP) to leucocyte and platelet- rich fibrin (L-PRF). Trends Biotechnol $2009 ; 27: 158-67$.

9- Saliba I: Hyaluronic acid fat graft myringoplasty: how we do it. Clinical Otolaryngology 2008; 33:607-628.

10- Gacek RR, Gacek MR. Anatomy of the auditory and vestibular systems. In: Ballenger JJ, Snow $\mathrm{JB}$, editors. Ballenger's otorhinolaryngology: head and neck surgery, Volume 1. 16th ed. Ontario: DC Becker Inc; 2003: p. 1-5.

11- Jellinge ME, Kristensen S, Larsen K.: Spontaneous closure of traumatic tympanic membrane perforations: observational study. J Laryngol Otol. 2015; 129:950-4.

12- Park MK, Kim KH, Lee JD and Lee BD: Repair of large traumatic tympanic membrane perforation with a Steri-strips patch. Otolaryngol Head Neck Surg. 2011; 145:581-5.

13- Lou Z and Wang Y: Evaluation of the optimum time for direct application of fibroblast growth factor to human traumatic tympanic membrane perforations. Growth Factors 2015; 33:65-70.

14- Gür ÖE, Ensari N, Öztürk MT, et al: Use of a platelet-rich fibrin membrane to repair traumatic tympanic membrane perforations: a comparative study. Acta Otolaryngol 2016; 136(10):10171023. 
15- Choukroun J, Adda F, Schoeffer C, Vervelle A. PRF: an opportunity in perio-implantology. Implantodontie. 2000;42:55-62.

16- Nuray Ensari, Özer Erdem Gür, Mehmet Türker Öztürk, Dinç Süren, Ömer Tarık Selçuk and Üstün Osma: The effect of platelet-rich fibrin membrane on the repair of perforated tympanic membrane: an experimental study, Acta OtoLaryngologica 2017, DOI: 10.1080/00016489.2017.1282169.

17- Habesoglu M, Habesoglu C, Sahin S, SahinYilmaz A, Korkmaz D, Tosun A and Karaaslan A: Platelet-Rich Fibrin Plays a Role on Healing of Acute Traumatic Ear Drum Perforation. The Journal of Craniofacial Surgery 2014; 25: 20562058.
18- Kumar RD: Application of Platelet Rich Fibrin Matrix to Repair Traumatic Tympanic Membrane Perforations: A Pilot Study. Indian J Otolaryngol Head Neck Surg (2017). https://doi.org/10.1007/s12070-017-1239-6

19- Nair NP, Alexander A, Abhishekh B, Hegde JS, Ganesan S and Saxena SK: Safety and Efficacy of Autologous Platelet-rich Fibrin on Graft Uptake in Myringoplasty: A Randomized Controlled Trial. Int Arch Otorhinolaryngol 2018, DOI https://doi.org/ 10.1055/s-00381649495.

20- Hosam M, Shaker M and Aboulwafa A: Effect of topical use of platelet-rich fibrin in repairing central tympanic membrane perforation using the endoscopic inlay butterfly cartilage myringoplasty technique. The Egyptian Journal of Otolaryngology 2017; 33:557-563

To cite this article: Mohamed F. Shindy, Ola S. El-Shimi , Mahmoud S. Belal , Abdelrahman A. Abdelalim. Use of platelet rich fibrin in acute traumatic tympanic membrane perforation compared with the conservative treatment. BMFJ 2020;37 (3): 512-523, DOI:10.21608/bmfj.2020.20388.1181 
Original article 